\title{
Investigation and Analysis on the Pathogen of Calf Diarrhea in Henan Province
}

\author{
Yan Lei ${ }^{1}$, Zhao Yuxi ${ }^{1,2}$, Liu Huan ${ }^{1}$, Yan Yuefei ${ }^{1}$, Ren Xiaoli ${ }^{1}$, Xue Yongkang ${ }^{1}$, Liu Xiaoman ${ }^{1}$, Zhang \\ Zhen $^{1 *}$
}

${ }^{1}$ Henan Dairy Herd Improvement Center, China

${ }^{2}$ School of Animal Science and Technology, Huazhong Agricultural University, China

\begin{abstract}
In order to understand the prevalence of various pathogens of calf diarrhea in Henan Province, C. parvum, BVDV, E. coli $\mathrm{K} 99, \mathrm{MAP}, \mathrm{BCoV}, \mathrm{BRV}$ antigens or antibodies were detected in feces and blood samples of 132 calves with diarrhea under 6 months in Henan Province by ELISA method. The results showed that the average positive rates of C. parvum, BVDV, E. coli K99, MAP, BCoV and BRV were 28.03\%, 6.82\%, 6.06\%, 5.03\%, 3.79\% and 2.27\%, respectively. The prevalence of $C$. parvum, BVDV, E. coli K99, MAP, BCoV and BRV exists in most areas of Henan Province, especially in central, southern and northern Henan. In addition to the single infection types of C. parvum, BVDV, E. coli K99, MAP, BCoV and BRV, there are also many kinds of mixed infection types. The proportion of single infection and mixed infection is 17:3. There are many types of mixed infection and the situation is complicated. The infection of MAP in diarrhea calves in Henan Province was reported for the first time. The results showed that there were a variety of single and mixed infection of calf diarrhea in Henan Province, and the single and mixed infection of $C$. parvum was more serious, which provided a reference for comprehensive prevention and control of calf diarrhea in Henan Province.
\end{abstract}

ABBREVIATIONS: CP: Cryptosporidium Parvum; EC: Escherichia Coli; MAP: Mycobacterium Paratuberculosis

\section{INTRODUCTION}

Calf diarrhea is a digestive tract disease of gastrointestinal dysfunction that often occurs in newborn young cattle, with dyspepsia, diarrhea, dysentery and other main symptoms [1], resulting in massive dehydration of calves, imbalance of water and salt metabolism of the body, auto poisoning and other metabolic syndrome [2], which is easy to cause poor growth and development, prolonged growth cycle, secondary infection and serious death of sick calves. It is also known as the "newborn calf killer" [3], which has caused huge economic losses to the rancher. There are many factors causing diarrhea in calves, which can be divided into infectious factors and non-infectious factors. Among the infectious factors, of Cryptosporidium parvum (C. parvum), of bovine viral diarrhea virus (BVDV), of bovine rotavirus (BRV), of bovine coronavirus (BCoV), Escherichia coli K99 (E. coli K99), which are widely studied and reported $[4,5,6]$.

\begin{tabular}{c|l} 
Quick Response Code: & $\begin{array}{l}\text { Address for correspondence: Zhang Zhen, Henan Dairy Herd Improvement } \\
\text { Center, China }\end{array}$ \\
\cline { 2 - 2 } & $\begin{array}{l}\text { Received: May 21, } 2020 \quad \text { Published: June 03, } 2020 \\
\text { How to cite this article: Yan L, Zhao Y, Liu H, Yan Y, Ren X, Xue Y, Liu X, Zhang Z. Investigation } \\
\text { and Analysis on the Pathogen of Calf Diarrhea in Henan Province. 2020 - 2(3) OAJBS.ID.000177. } \\
\text { DOI: } 10.38125 / O A J B S .000177\end{array}$ \\
\hline
\end{tabular}


Mycobacterium avium subsp. of Mycobacterium paratuberculosis. (MAP) is a pathogen causing chronic enteritis in ruminants, widely distributed all over the world, and causing significant economic losses [7], but there are few reports about calf MAP.

In order to understand the epidemic situation of the pathogen of calf diarrhea in Henan Province, the ELISA method was used to detect the prevalence of $C$. parvum, BRV, BCoV, E. coli K99, BVDV, $M A P$ antigen or antibody in calf feces and blood samples. The test results were statistically analyzed by region and infection type, so as to provide reference for the prevention and control of calf diarrhea in Henan Province.

\section{MATERIALS AND METHODS}

From 2018 to 2019, 132 faeces and corresponding blood samples of calves with obvious diarrhea symptoms were collected from large-scale farms in central, northern, southern, eastern and western Henan province (54 in central Henan, 36 in northern Henan, 22 in southern Henan, 11 in eastern Henan and 9 in western Henan).
Detection of $C$. parvum in fecal samples by ELISA (IDEXX, USA, P0063-1), detection of MAP in blood samples by ELISA (IDEXX, USA, P071305), detection of BVDV in blood samples by ELISA (IDEXX, USA, 99-43830), detection of BRV, BCoV E. coli K99 in fecal samples by triple antigen ELISA(IDEXX, USA, P0065-1), following the steps provided by the manufacturer. The detection results of 132 blood and fecal samples were counted, and the individual and mixed infections of six pathogens were analyzed.

\section{RESULTS AND ANALYSIS}

\section{ELISA Test Results}

The pathogen infection of C. parvum, BRV, BCoV, E. coli K99, BVDV and MAP in feces and blood samples of diarrhea calves from southern Henan, northern Henan, central Henan, western Henan and eastern Henan were detected by ELISA method. the positive infection rates were $28.03 \%, 6.82 \%, 6.06 \%, 5.03 \%, 3.79 \%$ and $2.27 \%$, respectively. The detection of pathogens in each area is shown in Figure 1.

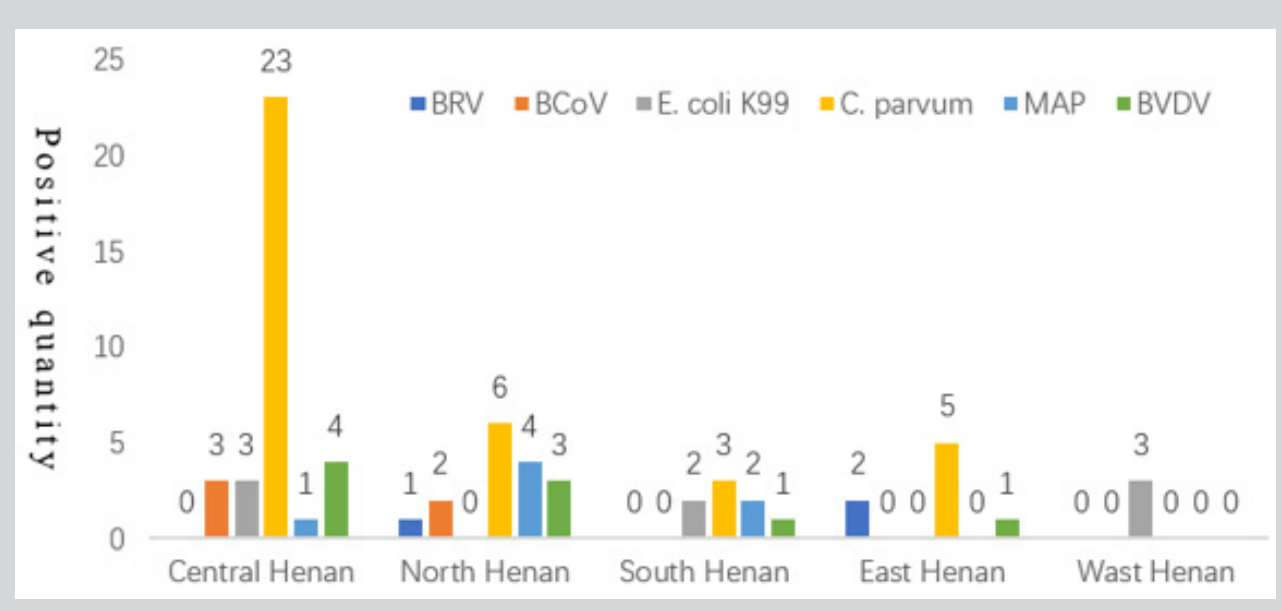

Figure 1: Distribution of calf diarrhoea pathogens in Henan Province.

A total of 37 C. parvum antigen positive samples were detected in feces, with an average positive rate of $28.03 \%$. C. parvum infection was found in central, southern, eastern and northern Henan, with the highest positive rate of $45.45 \%$ in eastern Henan and $42.59 \%$ in central Henan. A total of 9 blood BVDV antigen positive samples were detected, with an average positive rate of $6.82 \%$. There was an epidemic of BVDV in central, southern, eastern and northern Henan, with the highest positive infection rate of $9.09 \%$ in eastern Henan, followed by $8.33 \%$ in northern Henan. A total of 7 MAP antibody positive samples were detected in blood, with an average positive rate of 5.03\%. There was MAP infection in central, northern and southern Henan, with the highest positive infection rate of $11.11 \%$ in northern Henan, followed by $9.09 \%$ in southern Henan. A total of 8 E. coli $\mathrm{K} 99$ antigen positive samples were detected in feces, with an average positive rate of $6.06 \%$. E. coli K99 infection existed in central, southern and western Henan, with the highest positive infection rate of $33.33 \%$ (3/9) in western Henan, followed by $9.09 \%(2 / 22)$ in southern Henan. A total of $5 \mathrm{BCoV}$ antigen positive samples were detected in feces, with an average positive rate of $3.79 \%$. There was BCoV infection in central and northern Henan, and the positive infection rates in central and northern Henan were $5.56 \%$. A total of 3 stool samples with positive BRV antigen were detected, with an average positive rate of $2.27 \%$. There was an epidemic of BRV in eastern and northern Henan, with the highest positive infection rate of $18.18 \%$ (2/11) in eastern Henan, followed by $2.8 \%(1 / 36)$ in northern Henan.

\section{Analysis of Single and Mixed Infection}

The single infection and mixed infection of six pathogens $C$. parvum, BVDV, E. coli K99, MAP, BCoV and BRV were statistically analyzed in the feces and blood samples of calves with diarrhea. The results are shown in Table 1 . There is a single infection of six pathogens C. parvum, BVDV, E. coli K99, MAP, BCoV and BRV, among which the single infection rate of $C$. parvum is the highest, reaching $28.03 \%$, followed by BVDV, 6.82\%. In terms of mixed infection, there are five mixed infection types: BVDV / C. parvum, MAP / C. parvum, BRV / C. parvum, E. coli K99 / C. parvum and BVDV / E. coli K99. Among them, the mixed infection types of BVDV / C. parvum, MAP / C. parvum and E. coli K99 / C. parvum are more serious, and the infection rates are $15.79 \%, 15.79 \%$ and $10.53 \%$, respectively. The above results showed that the main causes of diarrhea in calves in Henan Province were single infection and mixed infection of six pathogens, C. parvum, BVDV, E. coli K99, MAP, BCoV and BRV. There were many types of mixed infection, and the situation was more complicated. The situation of single infection and mixed infection of $C$. parvum was more serious. 
Table 1: Statistical Analysis of single infection type and mixed infection type.

\begin{tabular}{|c|c|c|c|}
\hline Type & Detection Quantity & Positive Quantity & Positive Rate \\
\hline BVDV & 132 & 6 & $4.55 \%$ \\
\hline MAP & 132 & 4 & $21.97 \%$ \\
\hline C. parvum & 132 & 29 & $1.52 \%$ \\
\hline BRV & 132 & 2 & $3.79 \%$ \\
\hline BCoV & 132 & 5 & $3.79 \%$ \\
\hline E. coli K99 & 132 & 2 & $1.52 \%$ \\
\hline BVDV / C. parvum & 132 & 3 & $2.27 \%$ \\
\hline MAP / C. parvum & 132 & 1 & $0.76 \%$ \\
\hline BRV / C. parvum & 132 & 2 & $1.52 \%$ \\
\hline E. coli K99 / C. parvum & 132 & 1 & $0.76 \%$ \\
\hline BVDV / E. coli K99 & 132 & 2 & \\
\hline
\end{tabular}

\section{DISCUSSION}

In recent years, with the rapid development of cattle industry, calf diarrhea has been paid more and more attention by researchers at home and abroad, and a large number of investigations on the prevalence of pathogens related to bovine diarrhea have been carried out. Dai et al. [8] used the rapid detection kit to detect 55 calf diarrhea samples in Yangling City, China. It was found that the overall infection rate was Cryptosporidium 35.7\%, BRV 21.4\% E. coli $\mathrm{K} 99$ 21.4\%, BVDV 0.0\%, respectively. Wang et al. [9] isolated and identified $E$. coli of calf diarrhea in Hebei area. The results showed that more than $90 \%$ of, E. coli K99 was the main pathogenic bacteria. Song [10] isolated and identified E. coli from calves in some areas of Xinjiang and found that $E$. coli K99 was one of the main pathogens causing diarrhea and death of newborn calves in cattle farms. Zhang [11] used RT-PCR method to detect BRV in the feces of 91 calves with diarrhea in some areas of Xinjiang and found that the positive rate of rotavirus in each cattle farm was $23.08 \%$ 90.91\%. Zhao [12] used PCR method to investigate the pathogen of 147 calf diarrhea fecal samples collected in Henan Province. It was found that the positive rates of $C$. parvum, BRV and BCoV were $14.97 \%, 12.24 \%$ and $4.76 \%$, respectively. The positive rate of $E$. coli K99 was 2.04\%.

Lee et al. [13] detected a total of 14 pathogens in 207cases of diarrhea calves in Korea by PCR. The most common pathogens were BRV (34.8\%), E. coli (22.0\%), BVDV (8.5\%), BCoV (7.9\%) and Cryptosporidium (7.3\%). Yong et al. [14] used PCR method to investigate the prevalence of 11 intestinal pathogens in the feces of diarrhea calves from cattle farms in the Midwest of the United States, and multiple logical regression model was used to analyze the correlation between diarrhea and the detection of various pathogens. The results showed that more than $50 \%$ of the fecal samples of diarrhea calves contained a variety of pathogens. Statistical analysis showed that, BRV A group, BCoV, E. coli K99 and C. parvum were significantly correlated with calf diarrhea. Among them, C. parvum and BRV are the most common intestinal pathogens of calf diarrhea, with a high detection rate of $33.7 \%$ and $27.1 \%$, respectively.

In order to provide reference for comprehensive prevention and control of calf diarrhea in Henan Province, and to enrich the pathogenic epidemiological data of calf diarrhea in Henan Province, fecal and blood samples of 132 calves with obvious diarrhea symptoms were collected from 12 cities of Henan Province, and the related pathogenic antigens or antibodies were detected by ELISA method. The results showed that C. parvum, BVDV, E. coli K99, MAP, $\mathrm{BCoV}$ and BRV infection existed in most areas of Henan Province, and the average positive rates were $28.03 \%, 6.82 \%, 6.06 \%, 5.03 \%$, $3.79 \%$ and $2.27 \%$, respectively. C. parvum, BVDV, E. coli $\mathrm{K} 99$ and MAP infection were more serious, mainly single infection, and there were many types of mixed infection. The proportion of single infection and mixed infection was 17:3. Among them, the positive infection rate of $C$. parvum is higher than that of Zhao [12] in Henan, and lower than that of Yong [14] in the Midwest of the United States, which may be due to geographical factors, sampling quantity and different detection methods [15]. C. parvum is dominant in single infection and mixed infection, and it is easy for other pathogens to cause mixed infection, which may be due to the decrease of immunity of calves caused by $C$. parvum, which makes calves susceptible to other pathogens. It was found that the positive rate of $C$. parvum was the highest in single infection and mixed infection. So far, no specific drug has been developed to treat animal C. parvum [16], which also increases the difficulty of prevention and control of calf diarrhea in Henan Province.

MAP mainly affects domestic ruminants such as goats, sheep and cattle, causing chronic enteritis, which is one of the main pathogens of diarrhea in adult cattle [17] and causes serious economic losses to the dairy industry all over the world [18]. In the United States, the annual loss of the cattle industry affected by MAP is estimated to be about $\$ 1.5$ billion [19]. MAP infects a wide range of hosts, infecting wild animals as well as domestic ruminants. However, there are few studies on calves. In this experiment, the prevalence of MAP antibodies in the blood of calves with diarrhea in Henan Province was reported for the first time, and the total antibody positive rate was $5.03 \%$. Therefore, attention and detection of calf MAP should be strengthened.

\section{CONCLUSION}

Sum up the above, there are multiple pathogenic infections in calf diarrhea in Henan Province, with a single pathogen as the main infection, and $C$. parvum alone and mixed infection are serious. So, the attention of calf diarrhea in Henan Province should be strengthened, at the same time, the pathogen detection and 
surveillance of calf diarrhea should be strengthened, and the scope of pathogen detection should be expanded. The infected calves should be isolated and eliminated in time, and gradually establish and maintain disease-free herds, to achieve healthy breeding of dairy cows in Henan Province.

\section{REFERENCES}

1. Frank NA, Kaneene JB (1993) Management risk factors associated with calf diarrhoea in michigan dairy herds. Journal of Dairy Science 76(5): 1313-1323.

2. Ren WQ, Wang XZ (2010) Etiological analysis and treatment of calf diarrhoea. Chinese Animal Husbandry and Veterinary Medicine 37(10): 183-186.

3. Zhao J (2015) Laboratory diagnosis and comprehensive prevention and treatment of salmonella diarrhoea of calves in a dairy farm in Xianyang. North West Agriculture and Forestry University, China.

4. Al Mawly J, Grinberg A, Prattley D (2015) Risk factors for neonatal calf diarrhoea and enteropathogen shedding in New Zealand dairy farms. Veterinary Journal 203(2): 155-160.

5. Yong-Il C, Kyoung JY (2014) An overview of calf diarrhoea - infectious etiology, diagnosis, and intervention. Journal of Veterinary Science 15(1): 1-17.

6. Saif LJ, Smith KL (1985) Enteric viral infections of calves and passive immunity. Journal of Dairy Science 68(1): 206-228.

7. Motiwala AS, Amonsin A, Strother M (2004) Molecular epidemiology of Mycobacterium avium subsp. paratuberculosis Isolates Recovered from wild animal species. Journal of Clinical Microbiology, 42(4): 1703-1712.

8. Dai SH, Li C, Li Zhi Q (2018) Investigation on the main pathogens of 55 cases of calf diarrhoea in dairy farm. Progress in Veterinary Medicine 39(07): 134-136.
9. Wang MJ, Wen GY, Liu Y (2018) Isolation, identification, drug resistance and phylogenetic analysis of Escherichia coli causing calf diarrhoea in some areas of Hebei Province. Northern Animal Husbandry 564 (20): $22-23$.

10. Song K (2016) Isolation, identification and partial characteristics of Escherichia coli causing calf diarrhoea in Xinjiang. Shihezi University, China.

11. Zhang K (2016) Investigation on pathogens related to viral diarrhoea of calves in large-scale dairy farms in northern Xinjiang. Shihezi University, China.

12. Zhao YX (2018) Investigation of main pathogens of calf diarrhoea in Henan Province and verification of microfluidic chip of calf diarrhoea. Henan Agricultural University, China.

13. Lee S, Hwan K, Ha Y, Choi EW (2019) Causative agents and epidemiology of diarrhoea in Korean native calves. J Vet Sci 20: e64-76.

14. Cho YI, Han JI, Wang C (2013) Case control study of microbiological etiology associated with calf diarrhoea. Veterinary Microbiology 166(34): 375-385.

15. Xiao L (2010) Molecular epidemiology of cryptosporidiosis: An update. Experimental Parasitology 124(1): 80-89.

16. Murugkar, Sangeeta (2009) Pathogen detection using coherent antistokes. Raman Scattering Microscopy.

17. Chiodini RJ, Kruiningen HJV, Merkal RS (1984) Ruminant paratuberculosis (Johne's disease): The current status and future prospects. Cornell Veterinarian 74(3): 218-262.

18. Losinger, Willard C (2005) Economic impacts of reduced milk production associated with epidemiological risk factors for Johne's disease on dairy operations in the USA. Journal of Dairy Research 73(01): 33-43.

19. Stabel JR (1998) Johne's disease: a hidden threat. Journal of Dairy Science 81(1): 283-288. 